\title{
On Posner's second theorem in additively inverse semirings
}

\author{
Sara Shafiq* (D), Muhammad Aslam (D) \\ Department of Mathematics, Government College University, Lahore, Pakistan
}

\begin{abstract}
In this paper, we generalize Posner's second theorem in additively inverse semirings. This can be regarded as the generalization of Posner's theorem in semirings.
\end{abstract}

Mathematics Subject Classification (2010). 16Y60, 16W25

Keywords. additively inverse semirings, prime semirings, derivations

\section{Introduction}

Let $(S,+,$.$) be a semiring with commutative addition and an absorbing zero 0$. A semiring $S$ is called additively inverse semiring [6] if for every $a \in S$ there exists a unique

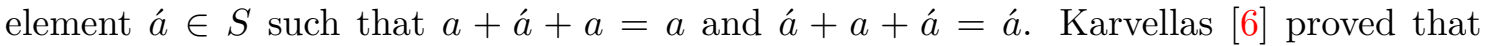
for all $a, b \in S,(a . b)^{\prime}=\dot{a} \cdot b=a . \dot{b}$ and $a \dot{a}=a b$. Throughout this paper, $S$ will represent an additively inverse semiring which satisfies the condition that for every $a \in S, a+a$ is in the center $Z(S)$ of $S$. This class of semirings is known as MA semiring [5]. In the last couple of years, MA semirings investigated and studied in the connection with certain additive maps (see [4,11-15]). By [5], a commutator [., .] in an additively inverse semiring defines as $[x, y]=x y+y x=x y+y \dot{x}$. We will make use of the commutator identities $[x y, z]=x[y, z]+[x, z] y,[x, y z]=[x, y] z+y[x, z]$ and $[x y, x]=x[y, x],[x, y x]=[x, y] x$ (see $[5,14]$, for their proof). $S$ is prime if $a S b=(0)$ implies either $a=0$ or $b=0$. It is easy to see that if $S$ is prime then the nonzero elements of $Z(S)$ are not zero divisors in $S$. An additive mapping $d: S \rightarrow S$ is called derivation if $d(a b)=d(a) b+a d(b), \forall a, b \in S$. Following [4], a mapping $F: S \rightarrow S$ is said to be commuting on $S$ if $[F(x), x]=0$ for all $x \in S$, and $F$ is said to be centralizing on $S$ if $[[F(x), x], S]=0$, holds for all $x \in S$.

A well-known theorem of E.C. Posner [10] says that a non-zero centralizing derivation of a prime ring $R$ forces $R$ to be commutative. This result has the virtue of being an important tool in ring theory, especially in the study of centralizing and commuting mappings and derivations. Posner's result has been generalized by many authors in several ways (see for example, [1,7-9, 16]). Recently, M.Anjum and M.Aslam [4] studied the notion of commuting mappings in MA semirings. In this paper, we shall attempt to generalize Posner's second theorem in additively inverse semirings.

\footnotetext{
*Corresponding Author.

Email addresses: saro_c18@yahoo.com (S. Shafiq), aslam298@hotmail.com (M. Aslam)

Received: 11.02.2017; Accepted: 05.02.2018
} 
We begin with the following useful lemma.

Lemma 1 ([14, Lemma 1.1]). Let $S$ be additively inverse semiring and $a, b \in S$. Then $a+b=0$ implies $a=\dot{b}$.

Lemma 2 ([4, Lemma 2.6]). Let $d$ be a derivation of prime additively inverse semiring $S$. If $a d(x)=0, x \in S$ then either $a=0$ or $d=0$.

Proposition 3 ([4, Theorem 2.7]). Let $S$ be prime and 2-torsion free, $d$ be a non-zero derivation of $S$. If $a \in S$ and $[d(x), a]=0, \forall x \in S$, then $a \in Z(S)$.

We also proved the following lemma in [12].

Lemma 4. Let $S$ be prime and 2-torsion free. If $a \in S$ such that $[a,[a, s]]=0, \forall s \in S$, then $[a, S]=0$.

Proof. Replacing $s$ by $s t, t \in S$ in $[a,[a, s]]=0$, we get, $[a, s][a, t]=0$. Replacing $t$ by $s t$ in the last expression, we have, $0=[a, s] s[a, t]$. But $S$ is prime so $[a, s]=0$.

Lemma 5. Let $S$ be 2-torsion free and prime. If $d_{1}, d_{2}$ are derivations of $S$ such that

$$
d_{1}(a) d_{2}(b)+d_{2}(a) d_{1}(b)=0, \forall a, b \in S,
$$

then one of $d_{1}, d_{2}$ is zero.

Proof. Replacing $a$ by $a d_{1}(c), c \in S$ in (1), we get

$$
d_{1}(a) d_{1}(c) d_{2}(b)+d_{2}(a) d_{1}(c) d_{1}(b)+a\left(d_{1}^{2}(c) d_{2}(b)+d_{2}\left(d_{1}(c)\right) d_{1}(b)\right)=0 .
$$

Replacing $a$ by $d_{1}(c)$ in (1) and using it in the last expression, we have,

$$
d_{1}(a) d_{1}(c) d_{2}(b)+d_{2}(a) d_{1}(c) d_{1}(b)=0, \forall a, b, c \in S .
$$

Applying Lemma 1 in (1), we have $d_{1}(c) d_{2}(b)=d_{2}(c) d_{1}(b)$, from this and (2), we obtain

$$
\left(d_{1}(a) d_{2}(c)+d_{2}(a) d_{1}(c)\right) d_{1}(b)=0 .
$$

By Lemma 2, either $d_{1}(a) d_{2}(c)+d_{2}(a) d_{1}(c)=0$ or $d_{1}=0$. If $d_{1}(a) d_{2}(c)+d_{2}(a) d_{1}(c)=0$ then we have $2 d_{2}(a) d_{1}(c)=0$ or $d_{2}(a) d_{1}(c)=0, \forall a, c \in S$. Replacing $a$ by $d_{2}(a)$, we have $d_{2}\left(d_{2}(a)\right) d_{1}(c)=0$. Again by Lemma 2 , either $d_{1}=0$ or $d_{2}(a)=0, \forall a \in S$. This completes the proof.

Theorem 6. Let $S$ be prime additively inverse semiring and d be a nonzero derivation of S such that

$$
[a, d(a)]=0, \forall a \in S
$$

Then $S$ is commutative.

Proof. By linearization of (3), we get

$$
a d(b)+d(b) \dot{a}+b d(a)+d(a) \dot{b}=0 .
$$

Thus we have,

$$
d(a b)+d(b) \dot{a}+b d(a)+2 d(a) \dot{b}=0 .
$$

From the given hypothesis, we have, $a d(a)+d(a) a+2 d(a) \dot{a}=0$ thus $d\left(a^{2}\right)=2 d(a) a$, by using Lemma 1. Replacing $b$ by $a x, x \in S$ in (4) and then using the last relation, we obtain $0=2 d(a) a x+a^{2} d(x)+d(a) x a ́ a d(x) \dot{a}+a x d(a)+2 d(a) a \dot{x}=a^{2} d(x)+d(a) x \dot{a}+a d(x) \dot{a}+$ $a x d(a)+2 d(a) a(x+\dot{x})=a^{2} d(x)+d(a) x a ́+a d(x) a+a x d(a)+2 a(x+\dot{x}) d(a)$. Because $a(x+x)$ is an additive idempotent so

$$
a^{2} d(x)+d(a) x a ́+a d(x) \dot{a}+a x d(a)=0 .
$$


Replacing $b$ by $x a$ in (4), we arrive at

$$
d(x) a^{2}+d(a) x a+a d(x) \dot{a}+a \dot{x} d(x)=0 .
$$

By Lemma 1, we have

$$
d(a) x a ́+a x d(x)=d(x) a^{2}+a d(x) a ́ .
$$

Using (6) in (5), we get

$$
a^{2} d(x)+2 a d(x) \dot{a}+d(x) a^{2}=0 .
$$

Thus we obtain, $a[a, d(x)]+[a, d(x)] \dot{a}=0$. Replacing $a$ by $a+d(x)$ in the last equation, we obtain

$$
d(x)[a, d(x)]+[a, d(x)] d(x)+[d(x), d(x)](a+\dot{a})=0 .
$$

But $[d(x), d(x)]\left(a+a^{\prime}\right)=d(x)(d(x)+d(x))(a+a)=d(x) d(x)\left(a+a^{\prime}\right)+d(x) d(x)(a+a)=$ $d(x) d(x)(a+a)$. Thus we have

$$
d(x)[a, d(x)]+[a, d(x)] d(x)^{\prime}+d(x) d(x)(a+a ́)=0 .
$$

This implies that

$$
d(x)[a, d(x)]+a d(x) d(x)^{\prime}+d(x) a d(x)+d(x) d(x)(a+a ́)=0 .
$$

As $(a+\dot{a}) \in Z(S)$ so we have,

$$
d(x)[a, d(x)]+a d(x) d(x)^{\prime}+d(x) a d(x)+\left(a+a^{\prime}\right) d(x) d(x)^{\prime}=0 .
$$

From this, we arrive at $[d(x),[a, d(x)]]=0$. Thus $[d(x), S]=0$, by Lemma 4 and therefore, by Proposition 3 , we conclude that $S$ is commutative.

Theorem 7. Let $S$ be prime and 2-torsion free d be a derivation of $S$ such that

$$
[[a, d(a)], S]=0, \forall a \in S
$$

then if $d \neq 0, S$ is commutative.

Proof. Linearization of (7) gives

$$
[[a, d(b)]+[b, d(a)], S]=0 .
$$

Replacing $b$ by $a x$ in the above equation, we have

$$
\Rightarrow[[a, a d(x)+d(a) x]+[a x, d(a)], S]=0 .
$$

Thus, we obtain

$$
\begin{gathered}
{[a[a, d(x)]+[a, d(a)] x+d(a)[a, x]+a[x, d(a)]+[a, d(a)] x, S]=0} \\
\Rightarrow\left[\left(a d(a)+d(a) a^{\prime}\right) x+d(a) x a^{\prime}+\operatorname{axd}(a), S\right]+\left[a^{2} d(x)+a d(x) a^{\prime}, S\right]=0 .
\end{gathered}
$$

Replacing $b$ by $x a$ in (8), we have, $[[a, d(x a)]+[x a, d(a)], S]=0$. From this, we get, $[[a, d(x) a]+[a, x d(a)]+x[a, d(a)]+[x, d(a)] a, S]=0$. Thus, we have

$$
\left[x\left(a d(a)+d(a) a^{\prime}\right)+d(x) a^{\prime 2}+d(a) x a^{\prime}+a d(x) a+\operatorname{axd}(a), S\right]=0 .
$$

From the given hypothesis, we have, $[a, d(a)] \in Z(S)$. Thus we obtain

$$
\begin{gathered}
{\left[\left(a d(a)+d(a) a^{\prime}\right) x+d(x) a^{\prime 2}+d(a) x a^{\prime}+a d(x) a+a x d(a), S\right]=0} \\
\Rightarrow\left[\left(a d(a)+d(a) a^{\prime}\right) x+d(a) x a^{\prime}+a x d(a), S\right]+\left[d(x) a^{\prime 2}+a d(x) a, S\right]=0 .
\end{gathered}
$$

By Lemma 1, we have

$$
\left[\left(a d(a)+d(a) a^{\prime}\right) x+d(a) x a^{\prime}+\operatorname{axd}(a), S\right]=\left[d(x) a^{2}+a d(x) a^{\prime}, S\right] .
$$

Combining (9) and (10), we obtain

$$
\left[a^{2} d(x)+d(x) a^{2}+2 a^{\prime} d(x) a, S\right]=0 .
$$

In particular, for $a \in S$, we have

$$
\left[a^{2} d(x)+d(x) a^{2}+2 a^{\prime} d(x) a, a\right]=0
$$




$$
\begin{gathered}
\Rightarrow a^{2} d(x) a+d(x) a^{3}+2 a ́ d(x) a^{2}+a^{\prime 3} d(x)+a^{\prime} d(x) a^{2}+2 a^{2} d(x) a=0 \\
\Rightarrow 3 a^{2} d(x) a+d(x) a^{3}+a^{\prime 3} d(x)+3 a^{\prime} d(x) a^{2}=0 .
\end{gathered}
$$

Replacing $x$ by $a$ in (11), we have

$$
d(a) a^{3}+a^{\prime 3} d(a)+3 a\left(d(a) a^{\prime}+a d(a)\right) a=0 .
$$

Thus we get

$$
d(a) a^{3}+a^{\prime 3} d(a)=3\left(d(a) a^{\prime}+a d(a)\right) a^{\prime 2} .
$$

Replacing $x$ by $a d(x)$ in (11), we get

$$
3 a^{2} d(a d(x)) a+d(a d(x)) a^{3}+a^{\prime 3} d(a d(x))+3 a^{\prime} d(a d(x)) a^{2}=0 .
$$

So, we have,

$$
\begin{aligned}
3 a^{2} d(a) d(x) a+3 a^{2} a d^{2}(x) a+d(a) d(x) a^{3}+a d^{2}(x) a^{3}+a^{\prime 3} d(a) d(x)+ \\
a^{\prime 3} a d^{2}(x)+3 a^{\prime} d(a) d(x) a^{2}+3 a^{\prime} a d^{2}(x) a^{2}=0 .
\end{aligned}
$$

Thus, we obtain

$$
\begin{aligned}
& a\left(3 a^{\prime} d^{2}(x) a^{2}+a^{\prime 3} d^{2}(x)+3 a^{2} d^{2}(x) a+d^{2}(x) a^{3}\right)+3 a^{2} d(a) d(x) a+ \\
& d(a) d(x) a^{3}+a^{\prime 3} d(a) d(x)+3 a^{\prime} d(a) d(x) a^{2}=0 .
\end{aligned}
$$

Replacing $x$ by $d(x)$ in (11) and using it in the last relation, we have

$$
3 a^{2} d(a) d(x) a+d(a) d(x) a^{3}+a^{\prime 3} d(a) d(x)+3 a^{\prime} d(a) d(x) a^{2}=0 .
$$

Pre-multiplying (11) by $d(a)$, we have

$$
3 d(a) a^{2} d(x) a+d(a) d(x) a^{3}+d(a) a^{\prime 3} d(x)+3 d(a) a^{\prime} d(x) a^{2}=0 .
$$

Applying Lemma 1 in the last equation to find value of $d(a) d(x) a^{3}$ and then using it in (13), we get

$$
\begin{gathered}
3 a^{2} d(a) d(x) a+3 d(a) a^{2} d(x) a^{\prime}+d(a) a^{3} d(x)+3 d(a) a d(x) a^{2}+a^{\prime 3} d(a) d(x)+3 a^{\prime} d(a) d(x) a^{2}=0 \\
\Rightarrow 3\left[a^{2}, d(a)\right] d(x) a+\left[d(a), a^{3}\right] d(x)+3[d(a), a] d(x) a^{2}=0 .
\end{gathered}
$$

In the view of (12), we obtain

$$
3\left[a^{2}, d(a)\right] d(x) a+3[a, d(a)] a^{\prime 2} d(x)+3[d(a), a] d(x) a^{2}=0 .
$$

By using the fact that $[d(a), a] \in Z(S)$, we have

$$
\left[a^{2}, d(a)\right]=a[a, d(a)]+[a, d(a)] a=2 a[a, d(a)] .
$$

Thus from (14), we have

$$
3[a, d(a)]\left(2 a d(x) a+a^{\prime 2} d(x)+d(x) a^{\prime 2}\right)=0 .
$$

But $S$ is prime and $[a, d(a)] \in Z(S)$, so by using the fact that center of $S$ is free of zero divisor, we have, either $[a, d(a)]=0$ or $2 a d(x) a+a^{\prime 2} d(x)+d(x) a^{\prime 2}=0$. If $[a, d(a)] \neq 0$, for some $a$ then for that $a$, we have

$$
2 a d(x) a+a^{\prime 2} d(x)+d(x) a^{\prime 2}=0, x \in S .
$$

Replacing $x$ by ax, we obtain

$$
2 a d(a) x a+a^{\prime 2} d(a) x+d(a) x a^{\prime 2}=0 .
$$

Replacing $x$ by $a$ in (15) and then post-multiplying by $x$ we get

$$
2 a d(a) a x+a^{\prime 2} d(a) x+d(a) a^{\prime 2} x=0 .
$$

By Lemma 1, (16) and (17), we arrive at

$$
2 a d(a)[x, a]+d(a)\left[x, a^{\prime 2}\right]=0 .
$$


Replacing $x$ by ax in (18), we get

$$
2 a d(a) a[x, a]+d(a) a\left[x, a^{\prime 2}\right]=0 .
$$

Pre-multiplying (18) by $a$, we get

$$
2 a^{2} d(a)[x, a]+a d(a)\left[x, a^{\prime 2}\right]=0 .
$$

Combining (19) and (20), we have

$$
[d(a), a]\left(2 a[x, a]+\left[x, a^{\prime 2}\right]\right)=0 .
$$

But $[d(a), a] \neq 0$, thus we have $2 a[x, a]+\left[x, a^{\prime 2}\right]=0$. From this, we have $2 a x a+2 a^{\prime 2} x+$ $x^{\prime} a^{2}+a^{2} x=0$ or $0=2 a x a^{\prime}+2 a^{2} x+x a^{2}+a^{\prime 2} x=2 a x a^{\prime}+x a^{2}+\left(2 a^{2} x+a^{\prime 2} x\right)=$ $2 a x a^{\prime}+x a^{2}+a^{2} x=a[a, x]+[a, x] a^{\prime}=[a,[a, x]]$. Thus $[a,[a, x]]=0$ so by Lemma 4 , $a \in Z(S)$. However, if $[d(a), a]=0, \forall a \in S$ then the required result follows from Theorem 6 .

Acknowledgment. We are thankful to the referee for his/her useful comments and suggestions.

\section{References}

[1] R. Awtar, Lie and Jordan Structure in Prime rings with derivations, Proc. Amer. Math. Soc. 41 (1), 67-74, 1973.

[2] H.J. Bandlet, M. Petrich, Subdirect products of rings and distrbutive lattics, Proc. Edin Math. Soc. 25, 135-171, 1982.

[3] J.S. Golan, The theory of semirings with applications in mathematics and theoretical computer science, John Wiley and Sons. Inc., New York, 1992.

[4] M.A. Javed and M. Aslam, Some Commutativity conditions in Prime MA-semirings, ARS Combinatoria 114, 373-384, 2014.

[5] M.A. Javed, M. Aslam and M. Hussain, On Condition $\left(A_{2}\right)$ of Bandlet and Petrich for inverse semirings, International Mathematical Forum 7, 2903-2914, 2012.

[6] P.H. Karvellas, Inversive semirings, J. Aust. Math. Soc. 18, 277-288, 1974.

[7] C.P. Lanski, Differential identities, Lie ideals, and Posner's theorems, Pacific J. Math. 134 (2), 275-297, 1988.

[8] L. Oukhtite, Posner's second theorem for Jordan ideals in Rings with involution, Expo. Math. 29, 415-419, 2011.

[9] L. Oukhtite, S. Salhi and L. Taoufiq, Commutativity conditions on Derivations and Lie ideals in $\sigma$-Prime Rings, Beiträge Algebra Geom. 51 (1), 275-282, 2010.

[10] E.C. Posner, Derivation in Prime Rings, Proc. Amer. Math. Soc. 8, 1093-1100, 1957.

[11] S. Sara and M. Aslam, Centralizers on semiprime MA semiring, Quasigroups Related Systems 24, 269-276, 2016.

[12] S. Sara and M. Aslam, On Lie ideals of Inverse semirings, accepted in Italian Journal of Pure and Applied Mathematics.

[13] S. Sara, M. Aslam and M.A. Javed, On Dependent elements and Free actions in Inverse semirings, International Mathematical Forum 11, 557 - 564, 2016.

[14] S. Sara, M.. Aslam and M.A. Javed, On Centralizer of semiprime inverse semirings, Discuss. Math. Gen. Algebra Appl. 36, 71-84, 2016.

[15] S. Sara, M. Aslam and M.A. Javed, On Jordan Mappings of inverse semirings, Open Math. 15, 1123-1131, 2017.

[16] J. Vukman, Commuting and Centralizing Mappings in Prime Rings, Proc. Amer. Math. Soc. 109 (I), 47- 52, 1990. 